\title{
Ultrafast Optical Properties of Dense Electron Gas in Silicon Nanostructures
}

\author{
A. Sieradzki • M. Basta $\cdot$ P. Scharoch $\cdot$ J.-Y. Bigot
}

Received: 30 October 2013 / Accepted: 2 December 2013 / Published online: 28 December 2013

(C) The Author(s) 2013. This article is published with open access at Springerlink.com

\begin{abstract}
We investigate the ultrafast dynamics of carriers in a silicon nanostructure by performing spectrally resolved femtosecond spectroscopy measurements with a supercontinuum probe. The nanostructure consists of a 158-nm-thick crystalline $\mathrm{Si}$ layer on top of which a $\mathrm{SiO}_{2}$ passivation layer leads to a very high quality of the Si surface. In addition, a dielectric function approach, including contributions from a Drude part and interband transitions, combined with the Transition Matrix Approximation is used to model the photogenerated carrier dynamics. The spectrotemporal reflectivity reveals two mechanisms. First, an electron-hole plasma is created by the pump pulse and lasts for a few picoseconds. Importantly, its spectral signature is either a positive or a negative change of reflectivity, depending on the probe wavelength. This is complementary to the already reported results obtained with degenerate frequency measurements. The second mechanism is a thermal diffusion of carriers which occurs during several hundreds of picoseconds. The overall dynamics at short and long delays in the whole visible spectrum is well explained with our model which shows that the main contribution to the reflectivity dynamics is due to the Drude dielectric function. The observation of this predominance of free carriers requires both a long lived high density of carriers as well as a little
\end{abstract}

A. Sieradzki $(\square) \cdot$ P. Scharoch

Institute of Physics, Wrocław University of Technology, Wybrzeże

Wyspiańskiego 27, 50-370 Wroclaw, Poland

e-mail: adam.sieradzki@pwr.wroc.pl

A. Sieradzki $\cdot$ J.-Y. Bigot

Institut de Physique et Chimie des Matériaux de Strasbourg, UMR

7504, CNRS, Université de Strasbourg, BP 43, 23 rue du Loess,

67034 Strasbourg Cedex 02, France

M. Basta

Department of Nanotechnology, Wroclaw Research Centre EiT+, ul Stabłowicka 147, 54-066 Wrocław, Poland influence of surface scattering as provided by our thin crystalline Si layer with passivated $\mathrm{Si} / \mathrm{SiO}_{2}$ interface.

Keywords Pump-probe spectroscopy · Carrier dynamics . Electron-hole plasma $\cdot$ Silicon nanostructure

\section{Introduction}

The developing technology of semiconductor devices at the nanoscale and the complexity of the devices themselves still requires to investigate the carrier dynamics on an increasingly shorter timescale and over a broad range of photon energies. In particular, for a device made of an active crystalline layer, the electronic and thermal properties depend not only on the layer itself but also on its surface or interface with a buffer layer. In the case of crystalline silicon, carriers can diffuse over a distance of $10 \mathrm{~nm}$ in $100 \mathrm{fs}$. Therefore, investigating the carrier properties in a Si layer of $\sim 100 \mathrm{~nm}$ requires simultaneously to generate and detect the carrier distributions on an ultrafast time scale and with a controlled surface, still compatible to technological requirements of ambient air pressure and temperature. The ideal situation, as implemented in the present work, is therefore to combine ultrafast pump-probe spectroscopy, using femtosecond laser pulses, with a wellsuited sample elaboration approach like the interface passivation in the case of Si.

The pump-probe femtosecond technique has been used over the past two decades [1-6] to investigate the photoexcited carrier dynamics in silicon. As it is well known [7], in a bulk semiconductor excited with a femtosecond optical pulse, as the system evolves toward equilibrium, there is a momentum and energy relaxation [8]. Momentum relaxation occurs on a few tens of femtoseconds scale via elastic and inelastic scattering [9]. On a similar time scale, the carrier-carrier scattering due to the Coulomb interaction leads to the thermalization of 
the electrons and holes to a Fermi-Dirac distribution [10]. Depending on the excess energy of the carriers on their respective conduction bands, this thermalization process is also assisted by carrier-phonon scattering. Meanwhile, additional processes specific to each considered semiconductor can take place, like the Auger recombination [11] or the intervalley scattering [12]. Importantly, the electrons and holes energy and momentum relaxation combined with their spatial diffusion result in a space and time dependent excited carrier density $N(r, t)$, with a typical time scale of the order of $10 \mathrm{ps.}$ During this time scale, the energy transfer to the lattice is sufficiently important so that its temperature $T(r, t)$ also depends on time and space via the diffusion of heat, within a time scale of a few hundreds of picoseconds. For modeling purposes, the two processes of carrier $N(r, t)$ dynamics and lattice temperature $T(r, t)$ diffusion can be considered, to a good approximation, as independent, regarding their initial values. In other words, it is reasonable to assume that the spatial temperature distribution left by excited carriers after they have relaxed forms an initial condition for the process of heat diffusion.

Interestingly, in the case of silicon, the carrier dynamics has been studied mostly from the point of view of high densities of carriers during the first picosecond. At low densities, the excited carriers form a nearly ideal gas of noninteracting particles. As the density raises up to $10^{22} \mathrm{~cm}^{-3}$, the carrier dynamics is well described by the theory of the electron-hole liquid (EHL) [13]. Moreover, at higher carrier densities $\left(2 \times 10^{23} \mathrm{~cm}^{-3}\right)$, a phase transition from a crystalline solid to a liquid phase may occur [14]. The regime of intermediate densities, where an electron-hole plasma is present, has also been studied [5-7], but so far, the overall dynamical regime from the plasma formation until the heat diffusion takes place has not been studied with details. In the present work, we investigate the carrier dynamics in silicon in a time scale covering both processes of carrier dynamics and heat diffusion, with densities high enough so that the initial excitation regime corresponds to the formation of an electron-hole plasma. Toward that purpose, and complementary to former works [5-7], we have performed time dependent reflectivity $\Delta R / R\left(\lambda, t, E_{\text {abs }}\right)$ measurements, as a function of wavelength in a broad band visible spectrum, for a long temporal scale (up to $800 \mathrm{ps}$ ), and as a function of absorbed pump density of energy $E_{\text {abs. }}$. The samples used are designed so that the electron-hole plasma is confined in a thin Si layer, with little influence of surface scattering effect. The complex observed spectrotemporal dynamics is well explained with a dielectric function that depends on $N(r, t)$ and $T(r, t)$, including both intraband and interband transition processes. Interestingly, our analysis shows that the dynamics is mostly affected by intraband processes corresponding to a Drude-like behavior of the semiconductor.

\section{Experiment}

Our study was conducted on a structure, shown in Fig. 1a, originating from the layered silicon wafer that was P-doped by implantation. The inhomogeneous $\mathrm{P}$ doping distribution varies by two orders of magnitude along the distance of $200 \mathrm{~nm}$ for implanted-diffused samples. The dopant density profile is shown in Fig. 1b. After the implantation process, the sample was subjected to the thermal oxidation (passivation), with the thickness of the passivation buffer layer equal to $\approx 200 \mathrm{~nm}$, to guarantee a good quality surface with the desired electronic properties. The passivation buffer layer was partially etched by reactive ion etching (RIE), leaving only $\mathrm{a} \approx 10$ nm-thick $\mathrm{SiO}_{2}$ layer. Next, another $\approx 117 \mathrm{~nm}$ of $\mathrm{SiO}_{2}$ was deposited on top of the 10-nm-thick $\mathrm{SiO}_{2}$ buffer layer. There is an amorphous silicon layer aSi in the structure, embedded at a depth of about $158 \mathrm{~nm}$ from the interface $\mathrm{cSi} / \mathrm{SiO}_{2}$ [15], where cSi denotes a crystalline silicon phase.

The time-resolved measurements of the carrier dynamics were carried out using a femtosecond pump-probe technique, in which a short pump pulse excites carriers and a timedelayed probe pulse measures the resulting change in reflectivity as a function of the pump-probe delay time. The optical pulses were generated using an amplified titanium:sapphire laser system operating at a repetition rate of $5 \mathrm{kHz}$. The pump beam, $150 \mathrm{fs}$ in duration, has a central frequency doubled at $397 \mathrm{~nm}$ (about $3.12 \mathrm{eV}$ ) using a beta barium borate (BBO)
Fig. 1 a Schematic view of the sample: $c S i$ crystalline $\mathrm{Si}$ and $a S i$ amorphous Si. b Shows the P doping distribution in the structure, as a function of the layer and depth measured by secondary ion mass spectroscopy
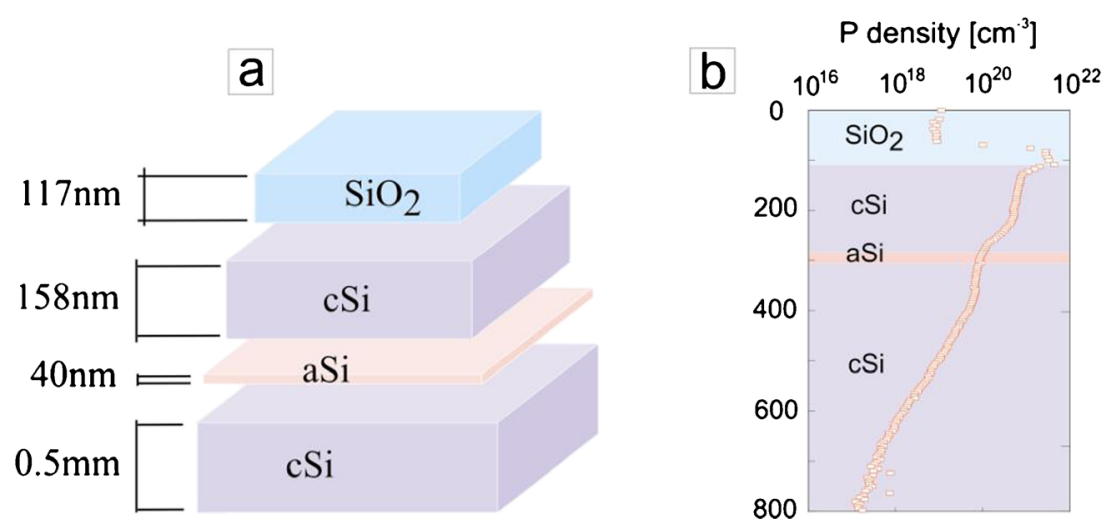
nonlinear crystal. The probe pulses with broad band spectrum were produced by femtosecond white light continuum generation on a thin sapphire plate. The probe beam reflected from the sample was dispersed by a grating in a monochromator and detected by a CCD camera. A band pass filter was placed in front of the detector in order to suppress all the side contributions from the pump and the fundamental beams. Because the supercontinuum pulse is chirped, the recorded reflectivity data have been time-corrected by performing a conformal mapping in the time frequency plane. To determine the chirp in the continuum pulse, we performed two-photon absorption on $\mathrm{ZnSe}$ using the same pump and probe pulses. Apart from the supercontinuum beam, we performed also single wavelength probe beam reflectivity measurements.

In both types of experiments, for constant absorbed energy $E_{\text {abs }}$ of the pump pulse, the reflectivity $R\left(t, \lambda, E_{\text {abs }}\right)$ of p-polarized probe pulses, with an angle of incidence on the sample of $20^{\circ}$, was measured as a function of time delay $t$ for chosen wavelengths $\lambda$. The changes in absorbed energy were achieved by varying the pump intensity. The quantity of interest is the dynamic differential reflectivity $(\Delta R / R)=\left[R\left(\lambda, E_{\mathrm{abs}}\right)-\right.$ $\left.R\left(\lambda, E_{\mathrm{abs}}=0\right)\right] / R\left(\lambda, E_{\mathrm{abs}}=0\right)$, where $R\left(\lambda, E_{\mathrm{abs}}=0\right)$ denotes the reflectivity of the unexcited sample. For measurements of the transient reflectivity, the resulting change in energy of the spectrally filtered probe beam was detected using lock-in amplifiers. For purposes of noise reduction, a separate reference beam was split off the probe beam prior to its interaction with the sample. To correct the pump-probe signal for intensity fluctuations in the continuum at the selected probe wavelength, the signal was normalized by the reference amplitude for each time delay, providing an accuracy of $3 \times 10^{-4}$. In this study, all the measurements were carried out at room temperature. Let us emphasize that the optical pumping remained below the melting threshold [16]. A precise control of the spot size of the pump beam (diameter $100 \mu \mathrm{m}$ ) on the sample during the measurements allowed for an accurate determination of the absorbed fluence. The maximum power of the beam was 1.4 $\mathrm{mJ} \mathrm{cm}{ }^{-2}$. Two time scales for the dynamical reflectivity are of main interest and named hereafter short ( $-1 \mathrm{ps} \rightarrow 5 \mathrm{ps})$ and long $(-20 \mathrm{ps} \rightarrow 400 \mathrm{ps})$ time behaviors.

\section{Results}

Figure 2 shows measured spectral differential reflectivity in the probe wavelengths range $490-710 \mathrm{~nm}$ for three delay times 5,100 , and $400 \mathrm{ps}$. The time dependent reflectivity spectrum is complex. For short delays (up to $30 \mathrm{ps}$ ), two regions can be distinguished. In the analyzed wavelength range, below $530 \mathrm{~nm}$ and above $610 \mathrm{~nm}$, a decrease in the reflectivity is observed, whereas in the range $530-610 \mathrm{~nm}$, the reflectivity change is distinctly positive. The position of the zero crossing points varies when changing the time delay. The

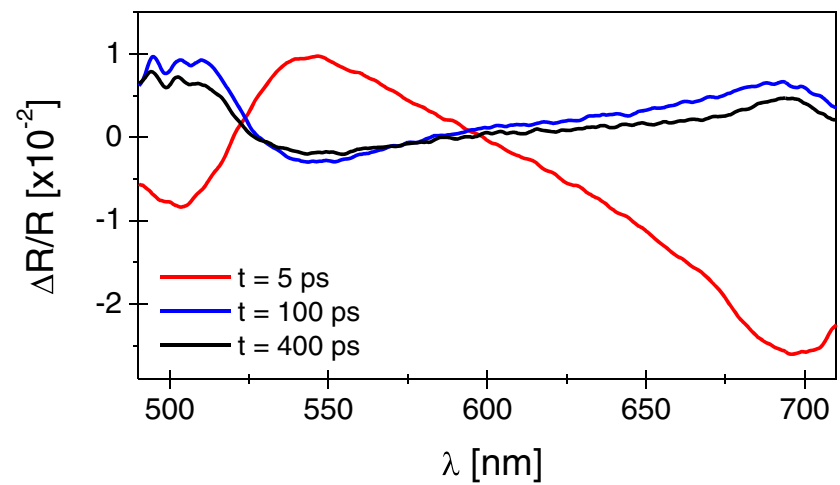

Fig. 2 Spectral variation of the differential reflectivity for three temporal delays

spectral range for which the differential reflectivity is positive spreads out reaching a maximum at less than $10 \mathrm{ps}$. The recovery time from the maximum negative change in reflectivity depends on the wavelength as discussed below in Fig. 3. After $80 \mathrm{ps}$, the spectral shape of the signal is changed so that for the energy range where the differential reflectivity $\Delta R / R$ was negative, it becomes positive and where $\Delta R / R$ was positive, it becomes slightly negative. At longer time delays, the recovery to the static value in the whole spectral range is observed.

Figure 3 shows both a short- and a long-time behavior of reflectivity following the pump pulse excitation for three chosen wavelengths. There are two distinct spectral regions, corresponding to the positive and negative reflectivity changes of $\Delta R / R$. For $\lambda$ out of the 530-610 $\mathrm{nm}$ range, the negative change in reflectivity $\Delta R / R$ recovers with different time constants until it changes sign, also at different times,
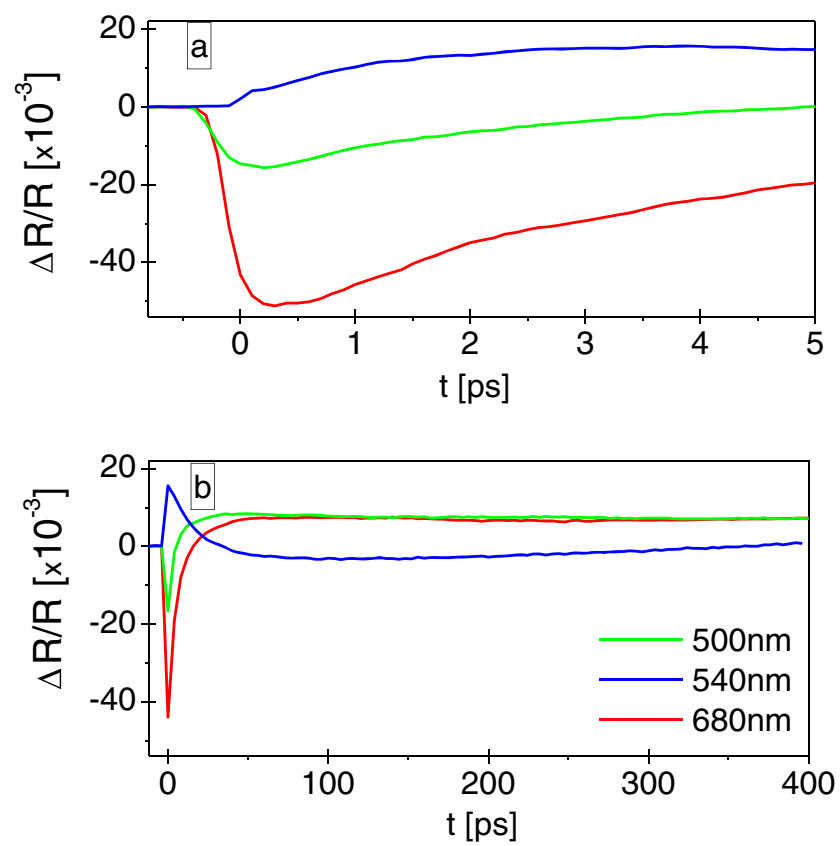

Fig. 3 Temporal variation of the differential reflectivity at short a and long b time delays for three different wavelengths 
depending on the wavelength (shorter times for shorter wavelengths). Afterwards, the positive $\Delta R / R$ slowly recovers toward equilibrium over several hundreds of picoseconds. For the wavelength $540 \mathrm{~nm}$, as well as in the spectral range $530-610 \mathrm{~nm}$ where $\Delta R / R$ is first positive, the differential reflectivity reaches a maximum in the first few picoseconds. It then decreases crossing the zero at $40 \mathrm{ps}$. At later time, $\Delta R / R$ slowly decreases and after $120 \mathrm{ps}$ it starts to recover. The time constants $\tau_{\mathrm{a}}$ as well as the zero crossing point are both wavelength dependent (not shown here).

\section{Discussion}

When discussing the transient reflectivity in the pump-probe experiment, one should be aware of various and complicated underlying physical mechanisms, so we start the discussion from some general notions. First, a high-energy laser pulse, partially reflected/scattered from the surface, excites the carriers with a given distribution in space, on a $\sim 100 \mathrm{fs}$ time scale. Then, the process of carrier energy scattering and recombination joined with diffusion takes place, over a few picosecond time, with a corresponding time space density distribution. The reflectivity in that time interval is affected mainly by the free intra and interband carrier absorption, including the $\Gamma \rightarrow X$ intervalley scattering, both limited by the Pauli blocking mechanism. Finally, after the excited carriers have been relaxed, the energy left and distributed in the form of heat begins to diffuse, resulting in a different time-space temperature distribution, with a characteristic time scale of a few hundred of picoseconds. For high-energy laser pulse, the temperature increase is sufficient to induce a significant increase in equilibrium densities, which in turn influences the reflection. Moreover, for certain sample architectures, the interferences of the probe beam may appear which modify the optical response and make the analysis even more complicated.

A common theoretical approach to discuss various factors determining the transient reflection is to expand to linear order the relative change in reflection coefficient with respect to the changes in refraction and extinction coefficients $[5,6,8]$. Then, the short-time (excited densities) and a long-time (temperature) effects are analyzed by considering the excited density and heat diffusion models. In this work, we propose a different approach, which is based on as adequate as possible numerical modeling and numerical experiments. We adopt the Drude-Lorenz model of the dielectric function $[17,18]$ :

$\varepsilon_{S}(\omega)=\varepsilon_{B}-\frac{\omega_{P}^{2}}{\omega(\omega+i \gamma)}+\sum_{j} \frac{A_{j}}{\left(\omega_{g j}^{2}-\omega^{2}\right)-i \Gamma_{j} \omega}$ in which the first term describes the background dielectric constant of Si crystal, the second term is the Drude term describing the metallic-like response from free carriers (optically or temperature induced), while the third term is the Lorentz oscillator linking the probability of absorption of a photon and subsequent transition of electron from valence to conduction band. We have applied an extended form of the Lorentz oscillator that allowed a precise treatment of the doping distribution and the resulting changes in the dielectric function. The meaning of the parameters is: $\omega$ and $\omega_{p}=\sqrt{N e^{2} / \varepsilon_{0} m_{e}^{*}}$ are the incident and plasma frequencies, $N$ is the density of carriers, $m_{\mathrm{e}}{ }^{*}=m_{\text {opt }} \cdot m_{e}-$ is the effective electronic mass, $m_{\mathrm{opt}}$ is the optical mass and $m_{e}$ is the mass of electron, $\varepsilon_{\mathrm{B}}-$ is the background dielectric function of silicon, $\gamma$ is the plasma damping corresponding to the inverse Drude damping time $\tau_{\mathrm{D}}, \omega_{g j}$ is interband transition frequencies, $\Gamma_{j}$ is the damping coefficient, and $A_{j}$-is the oscillator strength, and the summation in the third term is over the direct interband transitions. To take into account the interferences that appear in optical response of the structure presented in Fig. 1, we have used the one-dimensional Transition Matrix Approximation [18].

In the first step, we have simulated the static reflection coefficient, matching the experimental and simulation results, with an average deviation less than $1 \%$. For that purpose, we used the dopant density profile shown in Fig. $1 \mathrm{~b}$ to evaluate the equilibrium density distribution at room temperature. At $295 \mathrm{~K}$, in room temperature, for P-doped silicon up to concentrations $N_{d}=10^{18} \mathrm{~cm}^{-3}$, the dopants are fully activated. For higher doping concentration, only a fraction of the introduced $\mathrm{P}$ atoms are activated, and our evaluation shows that for the highest P concentration, reaching $N_{d} \sim 10^{21} \mathrm{~cm}^{-3}$, only a small fraction of phosphorous is active (about $6 \%$ ). This fact is represented by the active dopant concentration (Fig. 4). The equilibrium carriers density allowed us to evaluate the spatially varying dielectric function, taking into account also the amorphized layer and a part of the cSi substrate (Fig. 5). Subsequent calculation of absorption and refraction coefficient has led to the static value of the reflection coefficient which simulated spectral dependence agrees very well with the measurement (Fig. 6). In the simulation, the maximum value of the density in the distribution has been used as the fitting parameter, and for the curve shown in Fig. 6 the corresponding value is $N=10^{20} \mathrm{~cm}^{-3}$. The values of the other parameters are $m_{\mathrm{opt}}=0.36, \omega_{g 1}=5.1 \times 10^{15} \mathrm{~s}^{-1}, \omega_{g 2}=3.76 \times$ $10^{15} \mathrm{~s}^{-1}$, and $\omega_{g 3}=4.41 \times 10^{15} \mathrm{~s}^{-1}$. An interesting observation, both from experiment and from simulation, point of views, is that the interference pattern is present in the whole spectral range, which means that the penetration/observation depth of a probe beam is comparable with the cSi layer thickness (information is gathered from the whole $\mathrm{cSi}$ active layer).

In the next step, we focused on simulating $\Delta R / R$, for the wavelength range $490-720 \mathrm{~nm}$, with a spectral step of $2 \mathrm{~nm}$. 


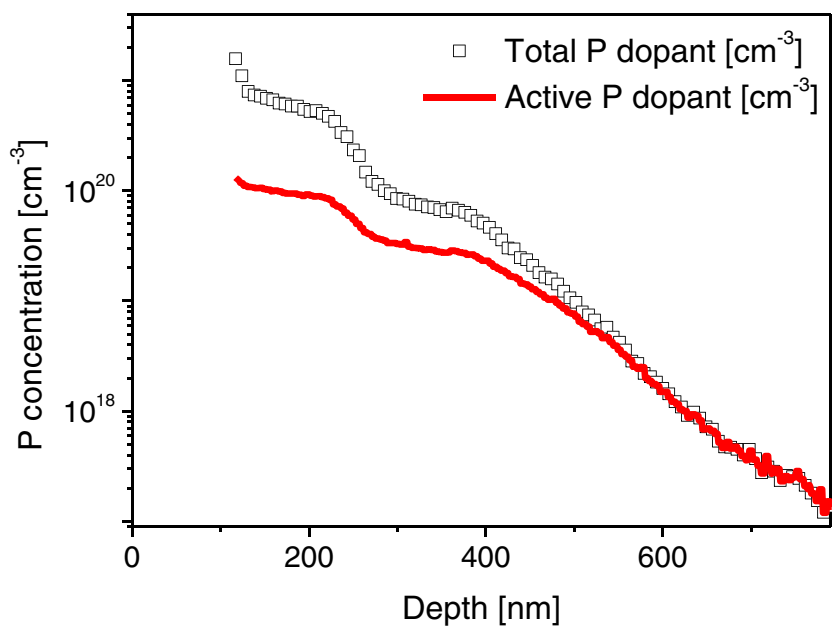

Fig. 4 Total vs active dopant concentration in the $\mathrm{cSi}$ layer

Here, as in works $[5,6,8]$, two different time scales have been considered. In a short-time scale (up to $20 \mathrm{ps}$ ), the simulation has been based on the assumption that the temporal free-carrier distribution plays a dominant role (thus, we discard here the Pauli blocking mechanism, which is not included in the model). The technique is then as follows: only the electronic density is varied while the other parameters are kept constant at their static values. The initial density distribution is assumed to coincide with the absorbed power distribution, calculated from the Poyinting vector and the absorption coefficient. The

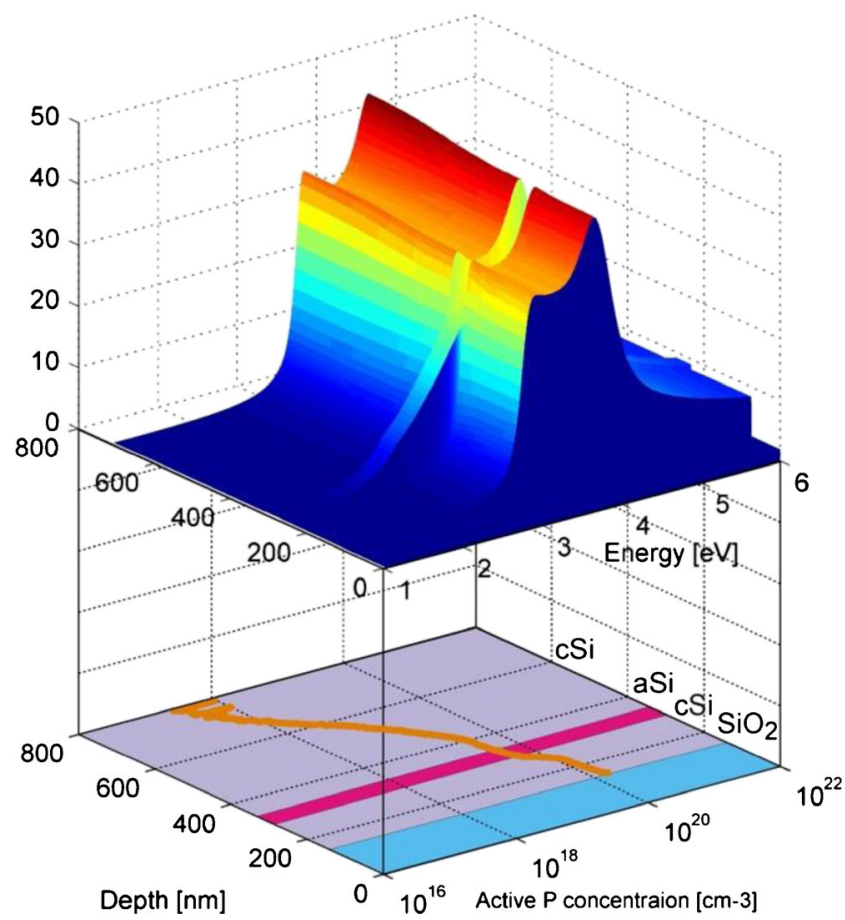

Fig. 5 Imaginary part of the dielectric function vs sample depth for accurate structure model (upper) and the active $\mathrm{P}$ distribution in each layer (lower). Thicknesses for each region in this model were $\mathrm{SiO}_{2}=$ $117 \mathrm{~nm}$, upper $\mathrm{cSi}=158 \mathrm{~nm}$, amorphized $\mathrm{Si}=40 \mathrm{~nm}$, and lower $\mathrm{cSi}=$ $400 \mathrm{~nm}$ (for precise doping simulation)

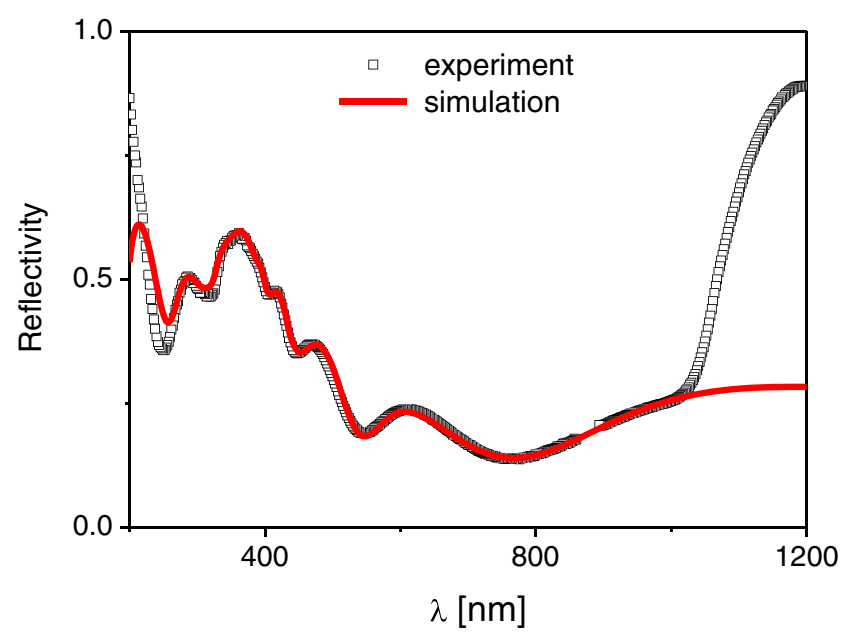

Fig. 6 Simulated vs measured static reflectivity

maximum value of the distribution is the fitting parameter. It is then assumed that during the time evolution, only the maximum value of the density changes and the shape of the profile is preserved. This assumption may seem a coarse one, but we performed many numerical experiments in which other distributions (e.g., Gaussian) have been tested and which showed that the average density rather than the particular shape of distribution affects the reflection. This is true also intuitively since the reflection, which varies with the absorption and refraction, carries an information that depends on the penetration depth of light. Therefore it is an averaging over specific spatial details and shape of the distribution. In our experiment, the penetration depth is comparable with the active cSi layer thickness, because the interference pattern is present. From this point of view, an exact modeling of carrier diffusions seems to be aimless.

The results of the simulation are shown in Fig. 7. The model reproduces quantitatively the observed spectral dynamics in the spectral range $490-570 \mathrm{~nm}$. An additional information obtained from the simulation is the time-resolved density, which enters the model as a single value parameter and thus can be considered as a kind of penetration depth dependent averaged density. This averaged density exhibits an exponential decay with the time constant $\tau \approx 10 \mathrm{ps}$, as shown in Fig. 8 .

The long-time behavior of reflectivity, as also discussed in $[5,6,8]$, is determined by the temperature increase. In our experiment, a simple estimation using the pump pulse energy, reflectivity, predicted absorption volume, and Si-specific heat shows that one can expect an average temperature increase of about $200 \mathrm{~K}$ and locally it can be higher. It is high enough to induce significant changes in the equilibrium density. For example, in intrinsic Si for a temperature change from 300 to $500 \mathrm{~K}$, the density increases from $1.5 \times 10$ to $9.2 \times$ $10^{19} \mathrm{~cm}^{-1}$, i.e., almost by 4 orders of magnitude. Here, we deal with the P-doped sample (n-type), with a given dopant density profile (Fig. 1b), and the room temperature value of 
Fig. 7 Comparison between experimental (solid lines) and simulation (broken lines) results of the differential reflectivity spectra for short delay times

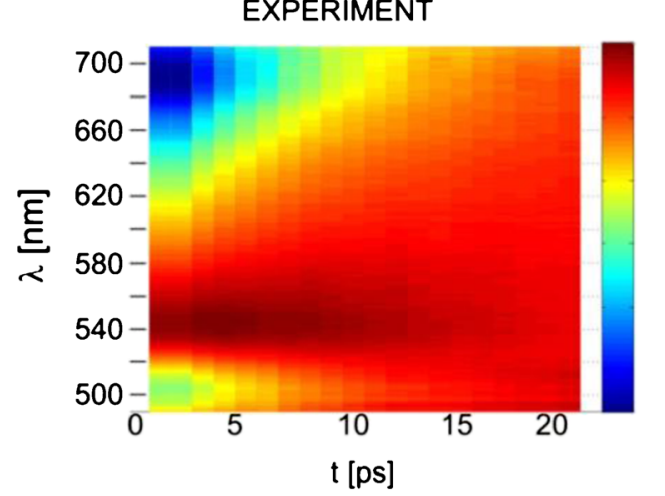

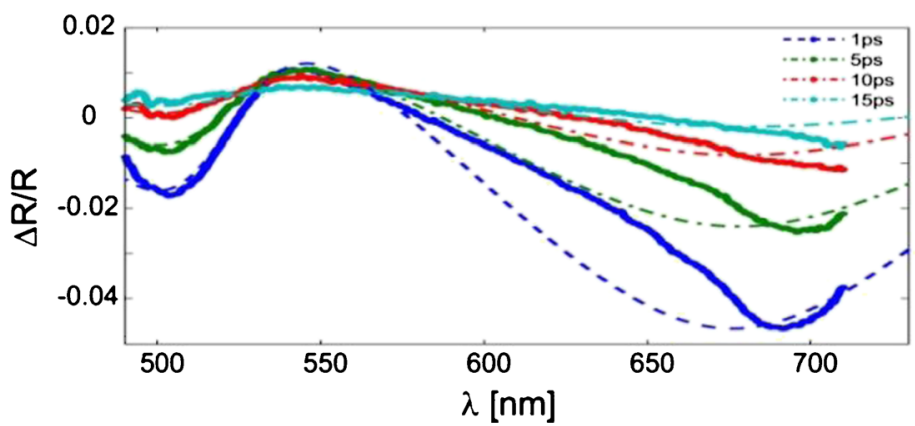

the excited density is plausible. In our model of optical response, we use the optical mass $m_{\text {opt }}$ to simulate the long-time behavior of reflectivity. We believe that at higher temperatures, the periodicity of the lattice potential is modified, which is reflected by the increase in the carrier optical mass $m_{\mathrm{opt}}$. Both parameters are equilibrium density dependent, but the average density itself is kept constant in the simulation, at the value of $N=10^{20} \mathrm{~cm}^{-3}$. This choice turned out to be sufficient to reproduce successfully the long-time reflectivity behavior. The results are presented in Fig. 9.

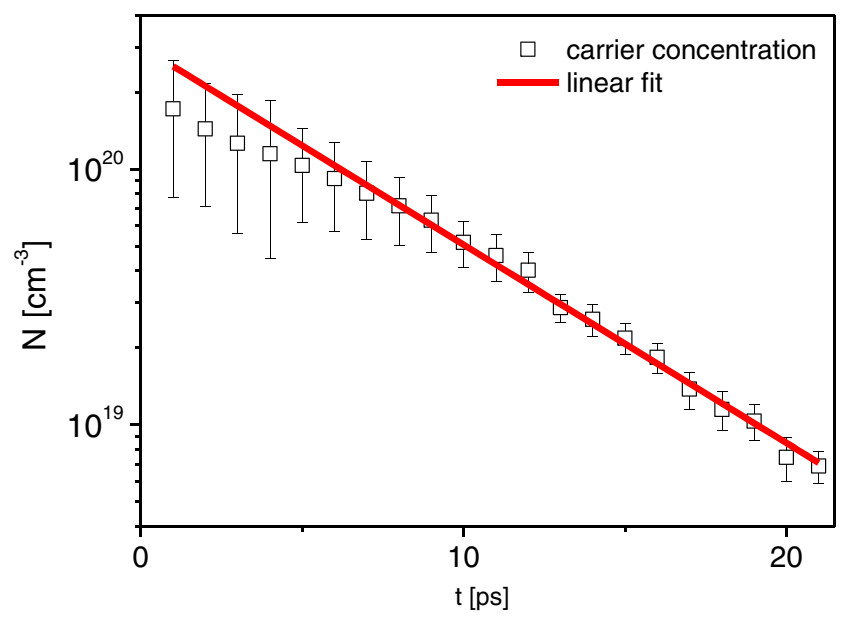

Fig. 8 Temporal dependence of the average carrier density used in the model of dielectric function to fit the experimental results. The fitting curve (red line) corresponds to an exponential decay
The optical mass increases three times more for the shortest time shown (100 ps), which is in agreement with other results published elsewhere [19].

In summary, one can conclude that the high excitation density is essential in the observed change of reflection, both in the short-time scale (high excited density) and in the longtime scale (high temperature increase). The high excitation densities are possible owing to the very good surface quality of our samples, achieved via special surface processing (passivation). The same experiment performed on a very

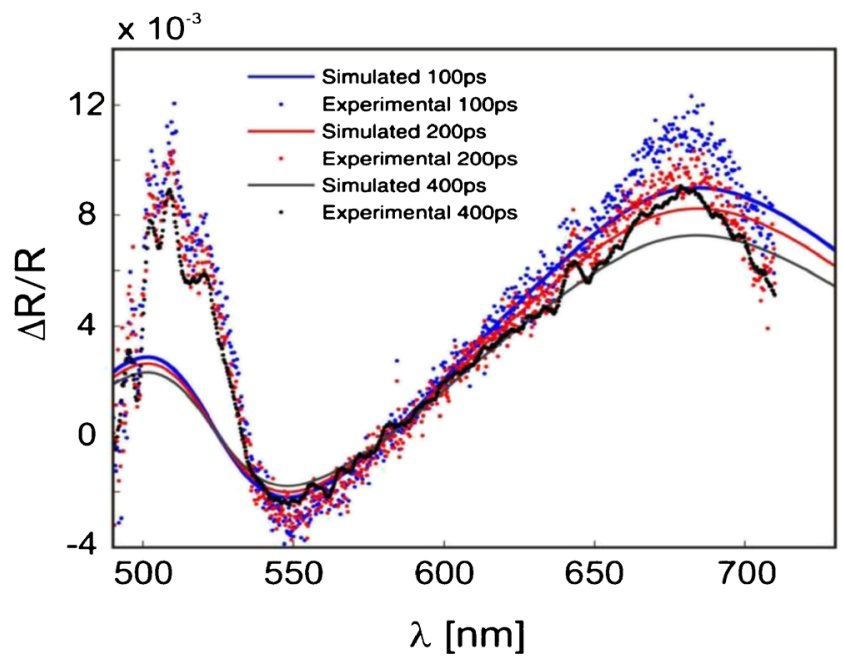

Fig. 9 Differential reflectivity spectra at longer time delays; comparison between experimental and simulation results. The fitting parameter is the optical mass $m_{\text {opt }}$. The electronic density is $N=10^{20} \mathrm{~cm}^{-3}$ 
similar sample but with the surface not passivated revealed a standard reflectivity behavior, i.e., the population bleaching mechanism leading to a decrease in the reflectivity.

\section{Conclusions}

The experiments performed in this work give an insight into the carrier dynamics, i.e., the excitation and relaxation processes in specially designed and preprocessed silicon nanostructures. The ultrafast, time, and spectrally resolved reflectivity measurements reveal important facts about the carriers behavior. At short delay times, the standard Pauli blocking mechanism is dominated by the quasimetallic reflection, whereas at longer times, the temperature effects (mainly via the free carriers) play a crucial role. However, the observed phenomena are possible only at high excitation densities; thus, the observations can be made only for a very high quality of illuminated interface, since otherwise the surface scattering and recombination processes make the generation of high densities impossible.

Acknowledgments The authors wish to thank prof. Z. T. Kuznicki for providing necessary silicon samples.

Open Access This article is distributed under the terms of the Creative Commons Attribution License which permits any use, distribution, and reproduction in any medium, provided the original author(s) and the source are credited.

\section{References}

1. Othonos A (1998) Probing ultrafast carrier and phonon dynamics in semiconductors. J Appl Phys 83:1789-1830

2. Sjodin T, Petek H, Dai H-L (1998) Ultrafast Carrier Dynamics in Silicon: A Two-Color Transient Reflection Grating Study on a 111 Surface. Phys Rev Lett 81:5664
3. Goldman JR, Prybyla JA (1994) Ultrafast dynamics of laser-excited electron distributions in silicon. Phys Rev Lett 72:1364-1367

4. Sokolowski-Tinten K, von der Linde D (2000) Generation of dense electron-hole plasmas in silicon. Phys Rev B 61:2643

5. Tanaka T, Harata A, Sawada T (1997) Subpicosecond surfacerestricted carrier and thermal dynamics by transient reflectivity measurements. J Appl Phys 82:4033-4038

6. Lioudakis E, Othonos A, Nassiopoulou AG (2006) Probing carrier dynamics in implanted and annealed polycrystalline silicon thin films using white light. Appl Phys Lett 88:181107

7. Shah J (1999) Ultrafast spectroscopy of semiconductors and semiconductor nanostructures. Springer, Berlin

8. Sabbah AJ, Riffe DM (2002) Femtosecond pump-probe reflectivity study of silicon carrier dynamics. Phys Rev B 66:165217

9. Portella MT, Bigot J-Y, Schoenlein RW, Cunningham JE, Shank CV (1992) k-space carrier dynamics in GaAs. Appl Phys Lett 60:21232125

10. Lioudakis E, Othonos A, Nassiopoulou AG, Lioutas CB, Frangis N (2007) Influence of grain size on ultrafast carrier dynamics in thin nanocrystalline silicon films. Appl Phys Lett 90:191114-191117

11. Hopkins PE, Barnat EV, Cruz-Campa JL, Grubbs RK, Okandan M, Nielson GN (2010) Excitation rate dependence of Auger recombination in silicon. J Appl Phys 107:053713

12. Bigot J-Y, Portella MT, Schoenlein RW, Cunningham JE, Shank CV (1990) Resonant intervalley scattering in GaAs. Phys Rev Lett 65: 3429-3432

13. Beni G, Rice TM (1978) Theory of electron-hole liquid in semiconductors. Phys Rev B 18:768-785

14. Sokolowski-Tinten K, Bialkowski J, von der Linde D (1995) Ultrafast laser-induced order-disorder transitions in semiconductors. Phys Rev B 51:14186-14198

15. Kuznicki ZT, Ley M (2003) Enhanced absorbance of a strained nanoscale Si-layered system. Appl Phys Lett 82:4241-4244

16. Sieradzki A, Kuznicki ZT (2013) Effects of carrier confinement and intervalley scattering on a photo-excited electron plasma in silicon, Plasmonics.. doi:10.1007/s11468-013-9582-2

17. Di Sia P, Dallacasa V (2011) Anomalous charge transport: a new "time domain" generalization of the Drude model. Plasmonics 6:99 104

18. Basta M, Kuznicki ZT, Sieradzki A (2011) Optical properties of crystalline and amorphous Si:P for device fabrication and structural modeling. Proc SPIE 8065:80650I

19. Riffe DM (2002) Temperature dependence of silicon carrier effective masses with application to femtosecond reflectivity measurements. J Opt Soc Am B 19:1092-1100 